\title{
Age differences in caloric-density preference as a function of strain of rats*
}

\author{
LEONARD F. JAKUBCZAK \\ Gerontological Psychology Laboratory \\ VA Hospital, St. Louis, Mo. 63125
}

When young rats, as well as humans, are presented with diets that differ in caloric density (kilocalories/gram), they prefer the calorically dense diet. How these preferences change with age is not known. A 2 by 2 by 3 by 7 experiment, representing strain, age, caloric-density difference, and day sets, was carried out in order to determine the influence of these factors on preference for calorically dense diets. The results indicated that, depending on the strain, old rats prefer calorically dense diets either to an equal degree or to a lesser degree than do young rats.

Do old rats prefer calorically dense diets to a greater degree than do young rats? One set of conditions known to affect the feeding of animals is the calorie content of the diet. When young rats, as well as humans, are presented with diets that differ in caloric density (kilocalories/gram), they prefer the calorically dense diet (Hamilton, 1964; Carlisle \& Stellar, 1969; Pilgrim \& Kamen, 1963). How these preferences change with age is not known. Furthermore, are these age differences in preference for calorically dense diets, if any, different for short- and long-lived strains of rats? Inbred strains of rats differ with respect to life expectancy (Robinson, 1965). This allows comparison of groups that are equal in age but differ with respect to residual life expectancy. Finally, are the age and/or strain differences in preference for the calorically dense diets, if any, due to differences in differential sensitivity to caloric density? Differences in preference for any substance may indicate differences in the sensitivity of the sensory apparatus (Robinson, 1965). The results of the present experiment indicate that, depending on the strain, old rats prefer calorically dense diets either to an equal degree or to a lesser degree than do young rats.

\section{METHOD}

The experiment was carried out according to a 2 by 2 by 3 by 7 mixed design (Case 2, Winer, 1971), representing strain, age, caloric-density difference, and day sets. The dependent variables were a preference index based on calories eaten (Klein \& DeFries, 1970) and body weight. $(\mathrm{N}=$ $71 ; \mathrm{Mij}=5-6$.)

The Ss were male rats from two strains: A x C 9935 (ACI/Mai, Microbiological Associates, Walkersville, Maryland) and Fischer 344 (Charles River Breeding Laboratory, Wilmington, Massachusetts). The former strain has a greater life expectancy than the latter strain (Robinson, 1965). Within each strain, the rats were either 187 days old or 745 days old on the first day of diet choice. The rats had been obtained at an age of

*This research was supported by 821 Funds of the Veterans Administration.
30 days and maintained under identical conditions in group cages (three per cage) until they reached the specific ages. Throughout their lives in this laboratory, the rats were fed Purina chow pellets and water ad lib. One month prior to the start of the present experiment, all the rats had completed participation in a single-stimulus calorie-tracking experiment (Jakubczak, unpublished). During the interim, the rats had been fed powdered chow from single cups.

Three diets were used in which $25 \%(\mathrm{w} / \mathrm{w})$ of petrolatum, U.S.P. Alba (Amojell, American Oil Co.), shortening (Crisco, Procter and Gamble Co.), or a mixture of the two substances ( $1.58 \mathrm{~g}$ petrolatum per $\mathrm{g}$ Crisco) added to powdered Purina chow yielded the following caloric densities (kilocalories/gram): 2.7, 5.1 , and $3.6 \mathrm{kcal}$, respectively. The texture (oiliness) of these diets was equal (Carlisle \& Stellar, 1969). The individually housed rats were given access to two loodcups, each containing one of the diets. All cups were systematically rotated as to position in the cage. Within each strain-age subgroup, the rats were assigned randomly to three caloric-density conditions (in kilocalories/gram). 2.7 vs $5.0 ; 2.7$ vs $3.6 ; 3.6$ vs 5.0 . The diet choices were presented for 21 days, and water was available ad lib. Each pair of cups and each rat were weighed daily.

The preference index was defined as 2 (preference ratio - 0.5 ) (Klein \& DeFries, 1970). The preference ratio was defined as the amount of the more dense diet eaten divided by the total amount eaten from both cups (in kilocalories). The data from the 21 days were reduced to seven day sets of three to expedite analysis. The analysis was carried out by means of a four-way unweighted means analysis of variance (Case 2, Winer,1971).

\section{RESULTS}

Figure 1 presents the preference index as a function of strain, age, and caloric-density difference, averaged across day sets. The analysis indicated a significant Strain by Age interaction ( $F=17.0$, df $=1$ and 59, $\mathrm{p}<.01$ ) that was independent of density difference and/or day sets. Thus, while the old rats of the Fischer strain preferred the calorically dense diet to a lesser degree than the young rats of that strain, there were no significant age differences in degree of preference among

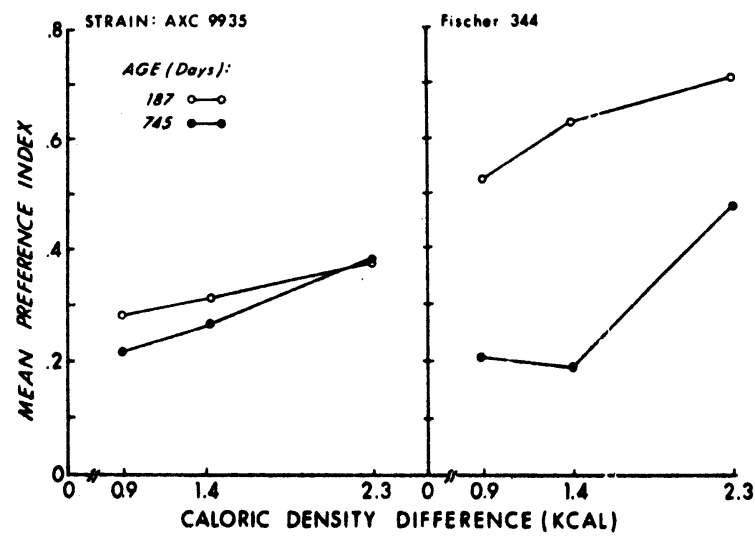

Fig. 1. Preference as a function of strain, age, and caloric-density difference, averaged across day sets. 


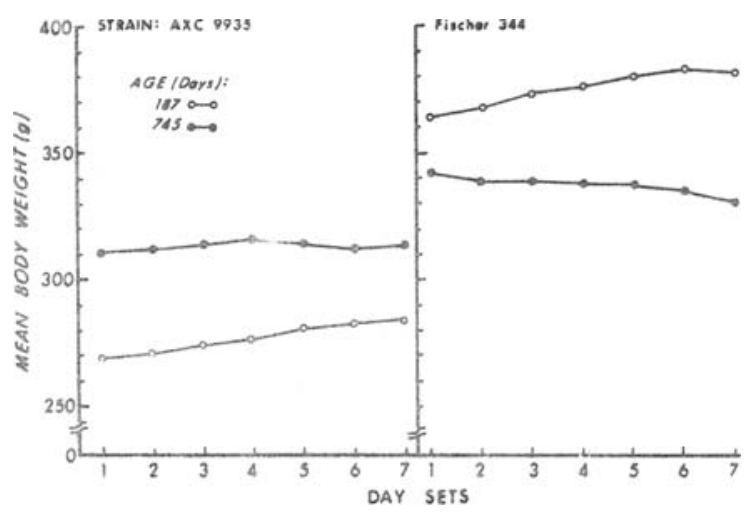

Fig. 2. Body weight as a function of strain, age, and day sets, averaged across caloric-density differences.

rats of the $\mathrm{AxC}$ strain. Furthermore, although the preference increased with increasing caloric-density differences $(F=8.34$, df $=2$ and $59, \mathrm{p}<.01)$, the increase was proportional across strain and/or age. The preference resulting from a difference of $2.3 \mathrm{kcal}$ was significantly greater than that resulting from either of the other density differences which did not differ between themselves. The analysis also indicated that preference increased with increasing day sets $(F=5.90$, $\mathrm{df}=6$ and $354, \mathrm{p}<.01$ ).

Figure 2 presents the effects of strain, age, and day sets on body weight, averaged across caloric-density differences. The analysis indicated a significant Strain by Age interaction ( $F=40.0$, df $=1$ and 59 , $\mathrm{p}<.01)$ and a significant Strain by Age by Day Set interaction $(F=3.97$, df $=6$ and $354, p<.01)$. Thus, while the old $\mathrm{A} \times \mathrm{C}$ rats weighed more than the young $\mathrm{A} \times \mathrm{C}$ rats, the old Fischer rats weighed less than the young Fischer rats. Also, the old A x C rats maintained their weights across day sets, while the old Fischer rats lost weight. The young rats of both strains gained weight across day sets equally. Finally, the effect of density difference on body weight, alone or in combination with the other factors, was not significant.

\section{DISCUSSION}

The results indicate that, depending on the strain, old rats prefer calorically dense diets either to an equal degree or to a lesser degree than do young rats. The higher degree of preference shown by the young Fischer rats than by the young $\mathrm{A} \times \mathrm{C}$ rats may reflect a strain difference with respect to weight, preference for fat as a thermoregulatory response, food conversion, basal metabolism, or any combination of these factors. The lower degree of preference shown by the old Fischer rats than by the young Fischer rats may reflect terminal changes in any of these factors, since weight loss is related to residual life expectancy (Everitt, 1957). Further research is needed to confirm and explain the strain and age differences in caloric-density preferences.

The results of this experiment are consistent with those of Hamilton (1967) and Carlisle \& Stellar (1969), who found preferences for high caloric densities and extend them to other strains and ages. The results also indicate the degree of differential sensitivity to caloric density or some factor correlated with density. The rat seems to be able to detect and respond to a difference in caloric density, or some factor correlated with density, of between 1.4 and $2.3 \mathrm{kcal}$. Since the differential sensitivity to caloric density was proportional across age and strain, at least within the range of densities tested, differences in sensitivity of the "sensory" apparatus cannot fully explain these age and/or strain differences in preference.

\section{REFERENCES}

Carlisle, H. J., \& Stellar, E. Caloric regulation and food preference in normal, hyperphagic, and aphagic rats. Journal of Comparative \& Physiological Psychology, 1969, 69, 107-114.

Everitt, $A$. The relation between body weight changes and life duration in male rats. Journal of Gerontology, 1957, 12, 128-135.

Hamilton, C. L. Rat's preference for high fat diets. Journal of Comparative \& Physiological Psychology, 1964, 58, 459-460.

Klein, T. W., \& DeFries, J. C. Taste sensitivity in infrahuman species: Use of a genetic model to test the validity of alternative measures. Behavior Research Methods \& Instrumentation, 1970, 2, 100-107.

Pilgrim, F. J., \& Kamen, J. M. Predictors of human food consumption. Science, 1963, $139,501-502$.

Robinson, R. Genetics of the Norway rat. New York: Pergamon Press, 1965.

Winer, B. J. Statistical principles of experimental design. (2nd ed.) New York: McGraw-Hill, 1971.

(Received for publication March 19, 1973.) 